\title{
Alcohol Use Disorder and The Heart
}

\author{
Ioannis Boutsikos and Lampros Samartzis* \\ School of Medicine, European University of Cyprus, Europe \\ *Corresponding author: Lampros Samartzis, School of Medicine, European University of Cyprus, Europe. \\ To Cite This Article: Ioannis Boutsikos, Lampros Samartzis. Alcohol Use Disorder and The Heart. Am J Biomed Sci \& Res. 2021 - 14(4). AJBSR. \\ MS.ID.002020. DOI: 10.34297/AJBSR.2021.14.002020.
}

Received: 㭗September 23, 2021; Published: 盋 October 27, 2021

\begin{abstract}
Background: Alcohol Use Disorder is related with heart morbidity. The aim of this review is to summarize effects of alcohol in the heart and to underline the relationship between alcohol consumption, drinking patterns and cardiovascular health.

Methods: We searched the databases PubMed and GoogleScholar by using the keywords "alcohol", "ethanol”, "disorder", "heart", "direct", "indirect", "effect", "detrimental", "beneficial", alone or in combination.

Results: Heart is affected by alcohol directly (protein synthesis-degradation dysregulation, increased oxidative stress, apoptosis, mitochondrial dysfunction, fibrosis of ECM) and indirectly (increased sympathetic nervous activity, renin-angiotensin-aldosterone system activation endothelial dysfunction, hypertension. Cardiovascular risk related to alcohol includes hypertension, metabolic syndrome, myocardial infarction, myocarditis, heart failure, arrhythmias, stroke. Drink pattern plays a key role in the phenotype of an alcohol-related disease.

Discussion-Conclusion: From a physician point of view, patients with alcohol use disorder need multidisciplinary approach to deal with all the concomitant conditions.
\end{abstract}

Keywords: Alcohol; Ethanol; Disorder; Heart; Direct; Indirect; Effect; Detrimental; Beneficial.

\section{Introduction}

Alcohol use has been one of the leading factors of the global burden of disease increasing the risk for different noncommunicable diseases, along with high fasting plasma glucose levels and obesity [1]. According to global statistics of World Health Organization on alcohol consumption, it is estimated a net CVD burden of 593000 deaths (3.3\% of all CVD deaths) and 13 million CVD DALYs (3.2\% of all CVD DALYs), and the average consumption of alcohol is highest in Europe with 8.71 liters and USA with 6.4 liters [2] Europeans and Australia citizens consume larger amounts of alcohol per capita in relation to other citizens around the globe ( 11.64 and 10.47 liters respectively) [3]. Use of alcohol and its effect on morbidity and mortality rates pose a substantial economic cost for the highand middle-income countries. The total healthcare cost (direct and indirect) accounts for approximately $2.5 \%$ of country's GDP [4].

Different studies have shown that the drinking patterns that the consumers follow could have substantial impact on their health. Heavy drinking and binge drinking in a time-specific limit (one day per week) has been correlated with higher risk for cardiovascular diseases. On the other hand, an evenly spread drinking pattern throughout a week led to decrease rates of cardiovascular events [5]. Thus, suggesting that the drinking pattern is very important in the alcohol-ischemic heart disease relationship, besides the amount of alcohol consumption.

Independently of its possible beneficial effects of alcohol on ischemic heart disease, its use should strictly be regulated in terms of public health policy because of its detrimental liver toxic effects and possible injuries that can cause, as well as and the fact that alcohol consumption is a subjective habit influenced by many social and personal factors [6]. The aim of this review is to highlight the relationship between alcohol consumption, drinking patterns and cardiovascular health.

\section{Methods}

We conducted a review of the literature on the PubMed and Google Scholar databases regarding the alcohol use disorder and its 
role on the heart. We used the keywords: alcohol, ethanol, disorder, heart, direct, indirect, effect, detrimental, beneficial.

\section{Results}

\section{Direct effects of alcohol in the heart}

Alcohol plays an important role affecting the myocardial function by impairing the cardiac protein synthesis. Chronic alcohol abuse as well as binge drinking dysregulates the balance of sarcoplasmic, reticular and myofibrillar protein synthesis and protein degradation with an overall net effect on catabolism in the myocardium. The effects of alcohol on protein synthesis is exerted by mTOR mainly via its phosphorylation which subsequently decreases the phosphorylation of 4E-binding protein 1 (4EBP1) and p70S6 kinase (p70S6K) [7]. On the other side concerning the increased degradation of proteins, chronic alcohol abuse increases the activity of ubiquitin-proteasome pathway (UPP). It is a major intracellular pathway, which is regulated by the expression of some specific target proteins such as atrogin-1 and muscle ring-finger ligase 1 (MuRF-1). Chronic alcohol abuse increases these specific proteins and promotes proteasome activity [8].

It has been shown that chronic alcohol abuse promotes cardiomyocyte apoptosis via oxidative stress. Both extrinsic and intrinsic apoptotic pathways are activated by alcohol because of the increasing activity of TNF-a and dysregulation of Bcl-2 and Bax proteins leading to shrinkage, nuclear condensation, DNA fragmentation and finally fragmentation into apoptotic bodies that are rapidly phagocytosed [9]. Alcohol and its metabolite, acetaldehyde, exert their toxic effects at the level of mitochondria which are the major contributors on the increased energy demands of the myocardium. This happens due to excessive oxidative stress that is promoted by alcohol [10]. Alcohol is metabolized by CYP2E1, which is a mitochondrial enzyme and generates Reactive Oxygen Species (ROS), increases superoxide production and decreasing membrane potential $[11,12]$.

\begin{tabular}{|c|}
\hline Table 1. \\
\hline Direct Effects of Alcohol on Heart \\
\hline Protein synthesis-degradation dysregulation \\
\hline Increased Oxidative Stress \\
\hline Apoptosis \\
\hline Mitochondrial Dysfunction \\
\hline Fibrosis of ECM \\
\hline
\end{tabular}

It has been hypothesized that cardiac dysfunction due to chronic alcohol abuse is also attributed its direct effect on myocardial fibrosis. More specifically, it has been shown that alcohol modifies the expression of cardiac Matric Metalloproteinases (MMPs) and Tissue Inhibitors of MMPs (TIMMPs) and thereby induce fibrosis of Extracellular Matrix (ECM). These changes in balance between
MMPs/TIMMPs are associated with excessive production of type I and III collagen leading to myocardial fibrosis [13] (Table 1).

\section{Indirect effects of alcohol in the heart}

Chronic alcohol abuse is associated with increased sympathetic nervous system (SNS) activity. The mechanism involves the suppression of the baroreceptor reflex and elevation of components of Renin-Angiotensin-Aldosterone System (RAAS), especially angiotensin II which has an excitatory effect on sympathetic nervous system [14]. Chronic activation of SNS has detrimental effects on cardiac muscle mainly due to promotion of excitationcontraction (E-C) coupling dysfunction and apoptotic pathways. Stress hormones such as norepinephrine have cardiotoxic effects leading to oxidative stress Increased SNS activity is related to the development of hypertension which in turn increases cardiac workload and impairs its diastolic function $[8,15]$. As it is mentioned above, RAAS activation happens because of increased diuresis which reduces the plasma volume. RAAS activation induces the sodium and water retention and thus increases the plasma volume and the preload of the heart [16]. Angiotensin II has direct effects on hypertrophy, apoptosis and ventricular remodeling thus leading to cardiac dysfunction [17]. Chronic alcohol use is associated with a rise in blood pressure, mainly the systolic, leading to the new term of alcoholic hypertension. Increased blood pressure is the result of the SNS activity and RAAS activation but also due to direct effects of alcohol on vascular wall, promoting vasoconstriction by increasing calcium vascular smooth muscle cells. Along with that, it has been shown that chronic alcohol consumption promotes oxidative stress at the vascular level causing endothelial dysfunction, inflammation and drop on the NO bioavailability [18] (Table 2).

\begin{tabular}{|c|}
\hline Table 2. \\
\hline Indirect Effects of Alcohol on Heart \\
\hline Increased Sympathetic Nervous Activity \\
\hline $\begin{array}{c}\text { Renin-Angiotensin-Aldosterone System activation Endothelial } \\
\text { Dysfunction }\end{array}$ \\
\hline Hypertension \\
\hline
\end{tabular}

\section{Cardiovascular risk related to alcohol}

World Health Organization states that there is no safe limit of drinking, and it appears that the safest measure is to not drink at all [2]. However, despite the difficulty of setting a safe threshold, multiple studies revealed that there is a close dose-response relationship between alcohol consumption and various diagnoses including:

\section{Hypertension}

A recent longitudinal study of people at ages 20 and 24 years and having their blood pressure measured at age of 24 , has shown that heavy alcohol consumption with a frequent pattern is strongly 
correlated with high blood pressure at these ages and tendency to develop hypertension [19]. Another meta-analysis of 36 trials with 2865 patients suggested that reduction of alcohol intake on individuals who drink $>2$ drinks/day is linked to further reduction of blood pressure levels along with corresponding CV events in a dose-dependent manner and importantly, with an apparent threshold effect [20]. The relationship between alcohol and hypertension is strong and it involves the aforementioned multiple mechanisms (either direct or indirect). Heavy drinkers should be encouraged to reduce the alcohol intake or on the other hand, nondrinkers should be encouraged to continue abstinence.

\section{Metabolic Syndrome}

In a study of 2088 Korean elderly patients, individuals with high alcohol intake had higher overall prevalence of metabolic syndrome comparing with patients with low alcohol consumption $(48.3 \%$ and $31.9 \%$ respectively) [21]. A meta-analysis of six prospective studies with a total of 28,862 participants suggested that light drinkers have lower risk than non-drinkers in developing metabolic syndrome (RR 0.86, 95\% CI: 0.75-0.99), while heavy drinkers have much higher risk (RR 1.84, 95\% CI: 1.34-2.52) [22]. Interestingly, it has been suggested that there is a correlation between appearance of metabolic syndrome and the timing of alcohol consuming or even the beverage type. Specifically, alcohol ingestion (most commonly wine) during mealtime is linked to lower risk for metabolic syndrome, whereas this risk appears higher when alcohol (most commonly beer) is ingested outside the scheduled meal plan [23].

\section{Myocardial infarction/Myocarditis/Heart Failure}

Heavy alcohol consumption is linked to higher prevalence of myocardial infarctions to individuals with established coronary artery disease, involving multiple pathways such as endothelial dysfunction, direct cardiac toxicity or destabilization of atherosclerotic plaques. A study showed that individuals with frequent hangovers showed 2-fold increase in death due to coronary artery disease [24]. As it is noted above, chronic alcohol use is related to direct cardiotoxic effects and consequent myocardial fibrosis affecting the overall cardiac function. Also, it is hypothesized that heavy drinking dysregulates the immune system balance and thus the individual is more susceptible to infections such as myocarditis, further deteriorating the cardiac function [25]. Chronic alcohol use with its direct and indirect effects on cardiovascular system leads to heart failure. Alcoholic cardiomyopathy is a relatively new term that includes the effects of alcohol on heart and the subsequently failing myocardium.

\section{Arrhythmias}

Another new term that describes the effect of alcohol on heart function is the "holiday heart syndrome" which refers to paroxysmal episodes of Atrial Fibrillation following weekends of binge drinking [26]. Also, there is an established relationship between heavy drinking and Sudden Cardiac Death (SCD) with an apparent increase of the incidences $>60 \%$ in relation to light drinkers. It is hypothesized that the malignant arrhythmias are a result of QT interval prolongation or electrolyte abnormalities or the deteriorating myocardial injury. Alcohol and its metabolites are powerful irritants of the myocardial cells leading to arrhythmias with possible fatal outcomes [27].

\section{Stroke}

Alcohol use disorder is a strong and independent risk factor for ischemic and hemorrhagic stroke. In a population-based Swedish registry, the risk of heavy drinkers for stroke is higher than lightdrinkers $(\mathrm{HR}=1.34, \mathrm{p}=0.02)$. Interestingly, the authors showed that there is an age-varying effect, meaning that the greater risk for stroke is in the range of 50-75 years old. Importantly, at this specific range heavy drinking is the most important risk factor for stroke [28]. A dose-response relationship is established between alcohol and hemorrhagic stroke showing that heavy drinkers and especially women have higher risk for hemorrhage than light drinkers [29]. Lastly, as noted above alcohol provokes episodes of Atrial Fibrillation which in turn could lead to cryptogenic strokes or large-area infarcts.

\section{The J-shaped curve theory}

Epidemiological evidence has shown that the correlation between alcohol intake and ischemic heart diseases follow a J-shaped curve. This suggests that light to moderate alcohol use is correlated with low risk for cardiovascular events, although as the amount of alcohol increases so does the risk. A recent metaanalysis of 84 cohort studies established a lower relative risk for cardiovascular events for drinkers than non-drinkers (relative risk 0.87). Interestingly, dose-response analysis revealed that the lowest point for CAD mortality occurred with 1-2 drinks per day and for stroke mortality with $<1$ drink per day [30]. Larsson et al published a meta-analysis of 8 prospective studies with a total of 202,378 patients comparing light/moderate drinkers to non-drinkers and showed that moderate drinking pattern has lower risk for heart failure (relative risk 0.85) [15]. The J-shaped curve theory is re-emphasized by more meta-analyses which suggested that all-cause cardiovascular mortality is lower on light drinkers compared to lifetime abstainers and supported by data concerning various cardiovascular biomarkers (higher levels of HDL and adiponectin, apolipoprotein) [31,32]. However, these studies have used as a baseline people that are non-drinkers who are not suitable for comparison with alcohol consumers because alcohol consumption varies over time and population leading to exaggeration of the beneficial effects on heart. In particular, there is 
evidence that the average amount of alcohol intake tends to decline with age due to health problems, mostly cardiovascular diseases, leading to accumulation of ill health and higher risk of mortality to non-drinkers [33]. Thus, there are no specific data to strongly urge individuals to start drinking even though they are life abstainers. These outcomes should be used as an additional motive for drinkers to limit their drinking into a light/moderate pattern [24] (Figure 1).

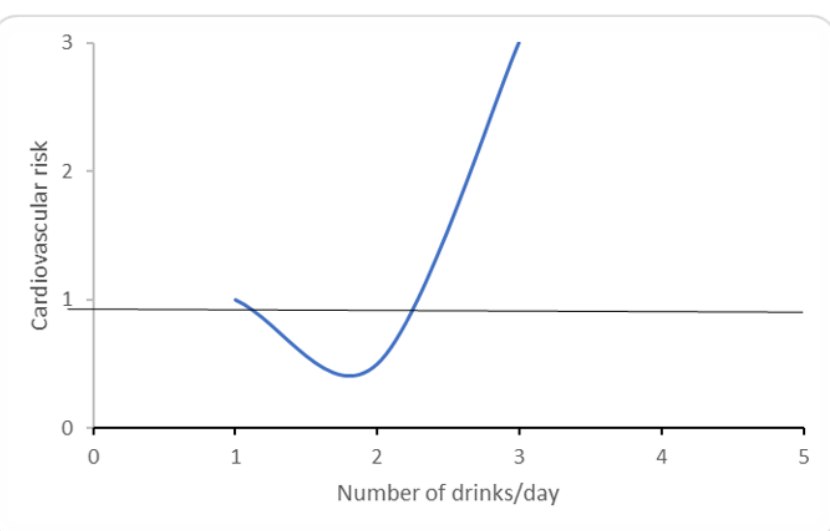

Figure 1: The J-shaped curve showing the relation between CVD risk and number of drinks per day. Light-moderate number of drinks per day decreases the risk for cardiovascular diseases comparing with the non-drinkers. However, the heavy alcohol use increases it significantly. (Black line shows the baseline of non-drinkers or abstinence).

\section{Conclusion}

Alcohol is the commonest substance of abuse and people are consuming it worldwide. The age varies tremendously and thus the detrimental effects of alcohol on public health is spreading in the community. However, a significant amount of evidence has shown that the drinking pattern plays a key role in the phenotype of an alcohol-related disease. Binge drinking and chronic heavy drinking pattern poses a significant risk for different diseases. Heart is affected by alcohol with many different mechanisms either direct or indirect. Alcohol has direct effects on the cardiomyocyte that impairs its function via decreasing oxidative phosphorylation and fibrosis leading to cardiac dysfunction. Also, it affects peripheral mechanisms such as RAAS which in turn affect the heart because of the abnormal loading conditions that are following. Excessive sympathetic discharge affects both the heart and the peripheral vascular bed leading to inflammation and adrenergic toxicity. Thus, alcohol use disorder is involved in many different heart diseases, and it always should be in the mind of the physician because alcohol use limitation would benefit patients.

It is important to state that alcohol use disorder is a public health problem with many aspects and different detrimental effects on population. Specific measures should be taken into national levels to reduce the burden of the disease. From a physician point of view, patients with alcohol use disorder need multidisciplinary approach to deal with all the concomitant conditions. Along with that, psychological or psychiatric support is always advised to this particular type of patients with regular follow-ups monitoring their mental health.

\section{Acknowledgement}

None

\section{Conflict of interest}

There is no conflict of interest.

\section{References}

1. Forouzanfar MH AA, Alexander LT (2016) Global, regional, and national comparative risk assessment of 79 behavioural, environmental and occupational, and metabolic risks or clusters of risks, 1990-2015: a systematic analysis for the global burden of disease study 2015. Lancet 388:1659-1724.

2. Hammer J, Parent M, Spiker D (2018) Global status report on alcohol and health 2018. Global status report on alcohol 65(1).

3. Peacock A, Leung J, Larney S, Colledge S, Hickman M, et al. (2018) Global statistics on alcohol, tobacco and illicit drug use: 2017 status report. Addiction 113(10): 1905-1926.

4. Rehm J, Mathers C, Svetlana Popova, Teerawattananon Y, Patra J (2009) Glob economic cost attributable to alcohol use and alcohol-use disorders. Lancet 373(9682): 2223-2233.

5. Ruidavets J, Ducimeti re P, Evans A, Montaye M, Haas B, et al. (2010) Patterns of alcohol consumption and ischaemic heart disease in culturally divergent countries: The prospective epidemiological study of myocardial infarction (PRIME). BMJ 341:c6077.

6. Roerecke M, Rehm J (2014) Alcohol consumption, drinking patterns, and ischemic heart disease: a narrative review of meta-analyses and a systematic review and meta-analysis of the impact of heavy drinking occasions on risk for moderate drinkers. BMC Med 12:182.

7. Vary TC, Lynch CJ, Lang CH (2001) Effects of chronic alcohol consumption on regulation of myocardial protein synthesis. Am J Physiol Heart Circ Physiol 281(3): H1242-H1251.

8. Gardner JD, Mouton AJ (2011) Alcohol effects on cardiac function. Compr Physiol 5(2): 791-802. 
9. Fernandez Sola J, Fatjo F, Sacanella E, Estruch R, Bosch X, Urbano Marquez A, et al. (2006) Evidence of apoptosis in alcoholic cardiomyopathy. Hum Pathol 37(8): 1100-1110.

10. Laurent D, Mathew J, Mitry M, Taft M, Force A, et al. (2014) Chronic ethanol consumption increases myocardial mitochondrial DNA mutations: A potential contribution by mitochondrial topoisomerases. Alcohol Alcohol 49(4): 381-389.

11. Hoek JB, Cahill A, Pastorino JG (2002) Alcohol and mitochondria: a dysfunctional relationship. Gastroenterology 122(7): 2049-2063.

12. Guo R, Ren J (2010) Alcohol dehydrogenase accentuates ethanolinduced myocardial dysfunction and mitochondrial damage in mice: role of mitochondrial death pathway. PloS One 5(1): e8757.

13. El Hajj EC, El Hajj MC, Voloshenyuk TG, Mouton AJ, Khoutorova E, et al. (2014) Alcohol Modulation of Cardiac Matrix Metalloproteinases (MMPs) and Tissue Inhibitors of MMP s Favors Collagen Accumulation. Alcohol Clin Exp Res 38(2): 448-456.

14. Triposkiadis F, Karayannis G, Giamouzis G, Skoularigis J, Louridas G, et al. (2009) The sympathetic nervous system in heart failure: physiology, pathophysiology, and clinical implications. J Am Coll Cardiol 54(19): 1747-1762.

15. Larsson SC, Wallin A, Wolk A (2018) Alcohol consumption and risk of heart failure: Meta-analysis of 13 prospective studies. Clin Nutr 37(4): 1247-1251

16. Sayer G, Bhat G (2014) The renin-angiotensin-aldosterone system and heart failure. Cardiol Clin 32(1): 21-32.

17. Rosenbaugh EG, Savalia KK, Manickam DS, Zimmerman MC (2013) Antioxidant-based therapies for angiotensin II-associated cardiovascular diseases. Am J Physiol Regul Integr Comp Physiol 304(11): R917-R928.

18. Shahbaz AU, Sun Y, Bhattacharya SK, Ahokas RA, Gerling IC, et al (2010) Fibrosis in hypertensive heart disease: molecular pathways and cardioprotective strategies. J hypertens 28(Suppl 1): S25-S32.

19. Wellman RJ, Vaughn JA, Sylvestre MP, O Loughlin EK, Dugas EN, et al (2016) Relationships between current and past binge drinking and systolic blood pressure in young adults. J Adolesc Health 58(3): 352-357.

20. Roerecke M, Kaczorowski J, Tobe SW, Gmel G, Hasan OS, et al. (2017) The effect of a reduction in alcohol consumption on blood pressure: a systematic review and meta-analysis. Lancet Public Health 2(2): e108-e120.
21. Kim JH, Ha JI, Park JM, Lee JS, Ahn AL, et al. (2018) Association of highrisk drinking with metabolic syndrome and its components in elderly Korean men: the Korean National Health and Nutrition Examination Survey 2010-2012. Korean J Fam Med 39(4): 233-238.

22. Sun K, Ren M, Liu D, Wang C, Yang C, et al. (2014) Alcohol consumption and risk of metabolic syndrome: a meta-analysis of prospective studies. Clin Nutr 33(4): 596-602.

23. Vieira BA, Luft VC, Schmidt MI, Chambless LE, Chor D, et al. (2016) Timing and type of alcohol consumption and the metabolic syndromeELSA-Brasil. PLoS One 11(9): e0163044.

24. Manolis TA, Manolis AA, Manolis AS (2019) Cardiovascular effects of alcohol: A double-edged sword/how to remain at the nadir point of the J-Curve? Alcohol 76: 117-129.

25. Maisch B (2016) Alcoholic cardiomyopathy. Herz 41(6): 484-493.

26. Tonelo D, Providência R, Gonçalves L (2013) Holiday heart syndrome revisited after 34 years. Arq Bras Cardiol 101(2): 183-189.

27. Perkiömäki J, Hookana E, Kaikkonen K, Junttila J, Kortelainen ML, et al. (2016) Blood alcohol in victims of sudden cardiac death in northern Finland. Europace 18(7): 1006-1009.

28. Kadlecová P, Andel R, Mikulík R, Handing EP, Pedersen NL (2015) Alcohol consumption at midlife and risk of stroke during 43 years of follow-up: cohort and twin analyses. Stroke. 46(3): 627-633.

29. Patra J, Taylor B, Irving H, Roerecke M, Baliunas D, et al. (2010) Alcohol consumption and the risk of morbidity and mortality for different stroke types-a systematic review and meta-analysis. BMC public health 10(1): $1-12$.

30. Ronksley PE, Brien SE, Turner BJ, Mukamal KJ, Ghali WA (2011) Association of alcohol consumption with selected cardiovascular disease outcomes: a systematic review and meta-analysis. Bmj 342: d671.

31.Xi B, Veeranki SP, Zhao M, Ma C, Yan Y, et al. (2017) Relationship of alcohol consumption to all-cause, cardiovascular, and cancer-related mortality in US adults. J Am Coll Cardiol 70(8): 913-922.

32. Huang Y, Li Y, Zheng S, Yang X, Wang T, et al. (2017) Moderate alcohol consumption and atherosclerosis: Meta-analysis of effects on lipids and inflammation. Wien Klin Wochenschr 129(21): 835-843.

33. Wannamethee SG, Shaper AG (1998) Alcohol, coronary heart disease and stroke: an examination of the J-shaped curve. Neuroepidemiology 17(6): 288-295. 